\title{
Status of the KM3NeT/ARCA telescope
}

\author{
Carla Distefano* for the KM3NeT Collaboration \\ Laboratori Nazionali del Sud, INFN, Catania, Italy \\ E-mail: distefano_celns.infn.it
}

The KM3NeT Collaboration has started the implementation of the ARCA detector, the next generation of the underwater kilometre cube scale neutrino telescope. The ARCA detector will be located in the Mediterranean Sea close to the Sicilian coast, about $100 \mathrm{~km}$ offshore Capo Passero at a depth of $3500 \mathrm{~m}$. This location will allow surveying a large part of the sky, including most of the Galactic Plane and the Galactic Centre. The first lines have been deployed and the analysis ongoing to validate the detector performance. We will present the status of KM3NeT/ARCA and the future prospects of the project.

EPS-HEP 2017, European Physical Society conference on High Energy Physics 5-12 July 2017

Venice, Italy

${ }^{*}$ Speaker. 


\section{Introduction}

The KM3NeT Collaboration is constructing a research infrastructure hosting the next generation underwater neutrino observatory. It will be distributed in two sites in the Mediterranean Sea with different configurations: ORCA (Mton scale 2500 depth offshore Toulon (France)) and ARCA (Gton scale, 3500 m offshore Capo Passero (Italy). The KM3NeT/ORCA detector, optimized to detect neutrinos of tens of $\mathrm{GeV}$, aims to determine the neutrino mass hierarchy. The KM3NeT/ARCA is the high-energy component and is dedicated for the search of extraterrestrial neutrino sources in the TeV-PeV range. The KM3NeT/ARCA detector is a three dimensional array of optical sensors detecting the Cherenkov light induced in water by the secondary charged particles produced in neutrino interactions. The arrival times of the light collected by optical sensors and their positions are used to reconstruct the secondary particles. From these the neutrino arrival directions can be inferred and their energies estimated. The main physics cases for KM3NeT/ARCA are the search for diffuse neutrino fluxes, in particular the investigation from a complementary field of view and with better angular resolution of the IceCube findings [1], and the search for point-like neutrino sources, with a particular focus on the galactic ones. The present status of the KM3NeT/ARCA detector and the future prospects of the project will be presented in this paper.

\section{The KM3NeT/ARCA neutrino telescope}

The basic unit of the detector is the Digital Optical Module (DOM) [2]. The DOM is a pressure-resistant glass sphere of 17-inch diameter containing 31 3-inch photomultiplier tubes (PMTs) with their associated electronics as well as the calibration instrumentation. Nineteen of these PMTs are located in the lower hemisphere, and are thus looking downwards, while 12 in the upper hemisphere are looking upwards (Fig.1). This novel design offers significant improvements with respect to optical modules with a single large-area PMT: the total photocathode area is about three times larger, the field of view covers almost the full solid angle, pixelisation allows for highpurity photon counting and directional sensitivity. All the necessary electronics for digitisation and data transmission is contained within each DOM.

A sequence of 18 DOMs, vertically spaced by $36 \mathrm{~m}$, forms a Detection Unit (DU) [2]. Two thin parallel ropes hold the DOMs in place. The DU is anchored on the seabed and kept taut by a submerged buoy at its top. A vertical "backbone" cable, running along the full length of the DU, provides connectivity for power and data transmission. This cable is an oil-filled plastic tube, in equi-pressure with sea water, containing electrical wires and optical fibres, with a breakout box at each DOM. A sketch of the DU is shown in Fig.1.

A set of 115 DUs, spaced with an average distance of $90 \mathrm{~m}$ and arranged with a roughly circular footprint, forms a "building block" (see Fig.1). The DUs are connected to junction boxes and finally to shore by means of a main electro-optical cable. The architecture is intrinsically modular allowing for a staged implementation of the telescope. For the deployment the DU is coiled on a spherical aluminum frame with $2 \mathrm{~m}$ diameter, called Launcher of Optical Modules (LOM). In this compact configuration the DU is deployed on the seabed and connected to the seafloor network. The LOM is then released, unfurling the DU while rotating upwards. It floats 


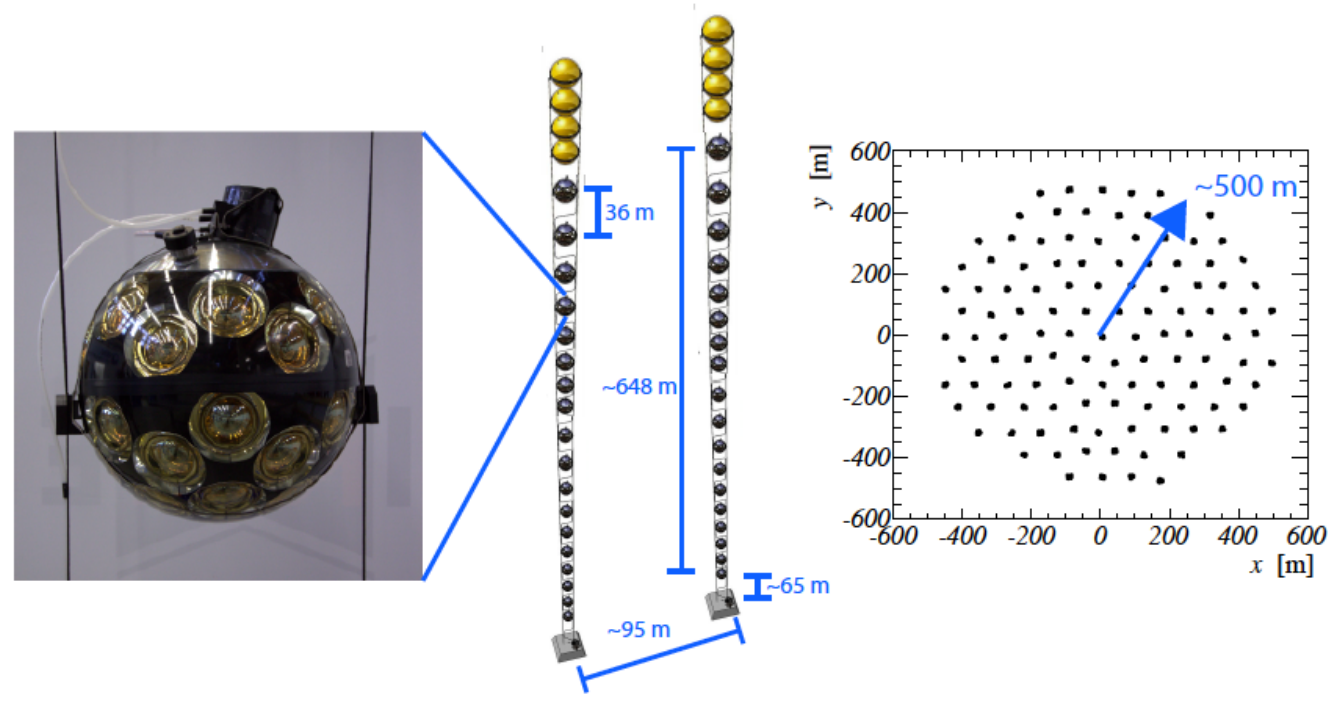

Figure 1: Schematic outline of a KM3NeT/ARCA building block.

to the surface where it is recovered for further use [2]. The ARCA telescope is currently under construction about $100 \mathrm{~km}$ off-shore Portopalo di Capo Passero (Sicily), at a depth of $3500 \mathrm{~m}$.

\section{The KM3NeT/ARCA Phase-1}

Following the successful prototyping phase [3, 4], the KM3NeT collaboration is pursuing the construction of the ARCA Phase-1. Consisting of 24 DUs, it will reach a volume of about 0.1 $\mathrm{km}^{3}$. ARCA Phase-1 will be the most sensitive neutrino telescope in the Northern hemisphere, with roughly 3 times the ANTARES sensitivity [5].

The first two strings were deployed in December 2015 and in May 2016 at the Italian site close to Capo Passero (Sicily) and at a depth of about $3500 \mathrm{~m}$. Data collected since their deployment were analysed to perform in-situ calibrations to determine the relative PMT efficiencies and PMTspecific transit times ('intra-DOM calibration') and to measure the DOM specific optical fibre lengths ('inter-DOM calibration') [6].

A measurement of the depth dependence of the coincidence rates induced by atmospheric muons looking at the coincidence multiplicity occurring in the different DOMs is on going [7]. Results for the two DUs are shown in Fig.2. The excellent agreement between the data and the Monte Carlo simulations confirms that the measured coincidence rate as a function of the depth matches the expected behaviour due to the depth dependence of the atmospheric muon flux.

\section{The KM3NeT/ARCA Phase-2.0}

The next objective will be the construction of KM3NeT/ARCA 2.0 at the Italian site. It will comprise two building blocks for a total instrumented volume of about $1 \mathrm{~km}^{3}$ and a sensitivity similar to that of IceCube. Full KM3NeT/ARCA construction, with six building blocks, is foreseen to proceed subsequently. 

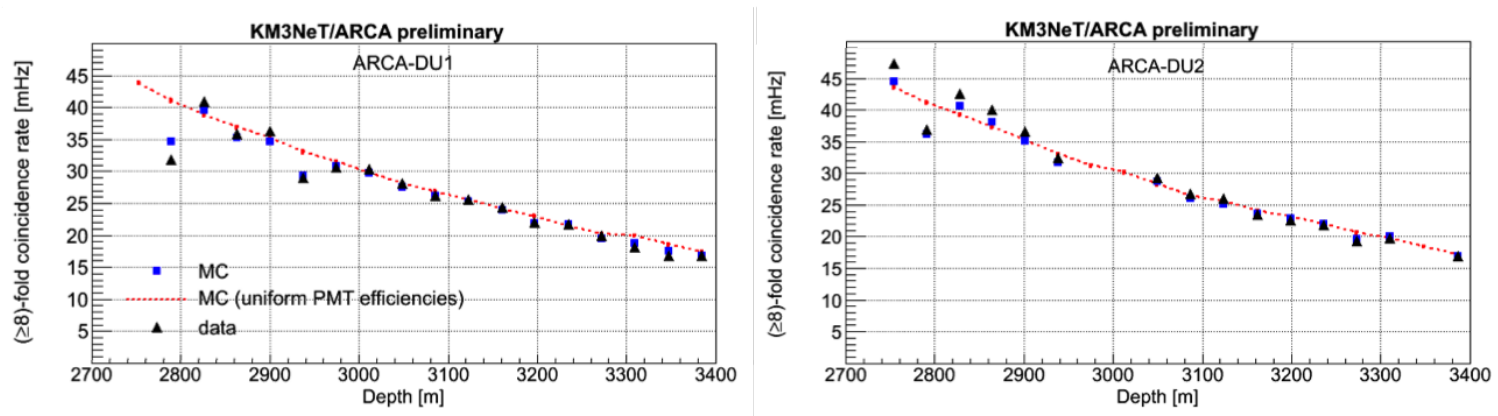

Figure 2: $\geq 8$-fold coincidence rate as a function of the depth for the two DUs. The red dashed lines show the base expectation from an ideal MC with uniform PMT efficiencies which neglects the differences between individual PMTs as determined from in-situ calibration. The black points show the data. The blue points show the normal full MC data where PMT efficiencies have been taken into account.

Evaluation of the KM3NeT/ARCA 2.0 telescope performance has been carried out using Monte Carlo simulations including the neutrino interaction in the medium, the propagation of the resulting secondary particles, the Cherenkov light generation and propagation in water and the detector response. The optical water properties measured at the Capo Passero site have been used [8]. Background due to the presence of $40 \mathrm{~K}$ in salt water was simulated adding an uncorrelated hit rate of $5 \mathrm{kHz}$ per PMT plus higher-fold coincidence rates as determined by GEANT simulations and in agreement with the results from the prototype optical modules. Backgrounds due to down-going atmospheric muons and to neutrinos originating in cosmic-ray interactions in the atmosphere have also been taken into account in the simulations.

KM3NeT/ARCA is sensitive to all neutrino flavours, since events of different topology (tracklike and shower-like events) can be detected and identified. An event reconstruction technique that fully exploits the DOM capabilities has been developed [9]. A very good angular resolution of about $0.2^{\circ}$ (left panel in Fig. 3) and an energy resolution of $27 \%$ in $\log \left(\mathrm{E}_{\mu}\right)$ are achieved for neutrino energies above $10 \mathrm{TeV}$. For high-energy events $(\mathrm{E}>50 \mathrm{TeV})$ the shower reconstruction reaches an accuracy on the vertex reconstruction of about $1 \mathrm{~m}$, an angular resolution of $2^{\circ}$ and an energy resolution of roughly $10 \%$.

Monte Carlo simulations indicate that KM3NeT can observe the expected high energy diffuse neutrino fluxes with a high significance with few year of observation [10]. Besides the discovery flux and the sensitivity to point-like sources (right panel in Fig. 3) will be more than one order of magnitude better than the IceCube ones in the Southern hemisphere and comparable to it elsewhere, assuming the same exposure [11].

\section{Conclusions}

The construction of the KM3NeT/ARCA detector, which in its final configuration consists of 2 building blocks of 115 DUs with a total instrumented volume of about $1 \mathrm{~km}^{3}$, has begun. KM3NeT/ARCA Phase-1 is fully funded and will consist of 24 strings at the Italian site near Capo Passero (Sicily). The first two DUs were deployed and collected data analysed to perform the detector calibration. A measurement of the depth dependence of the coincidence rates induced by 

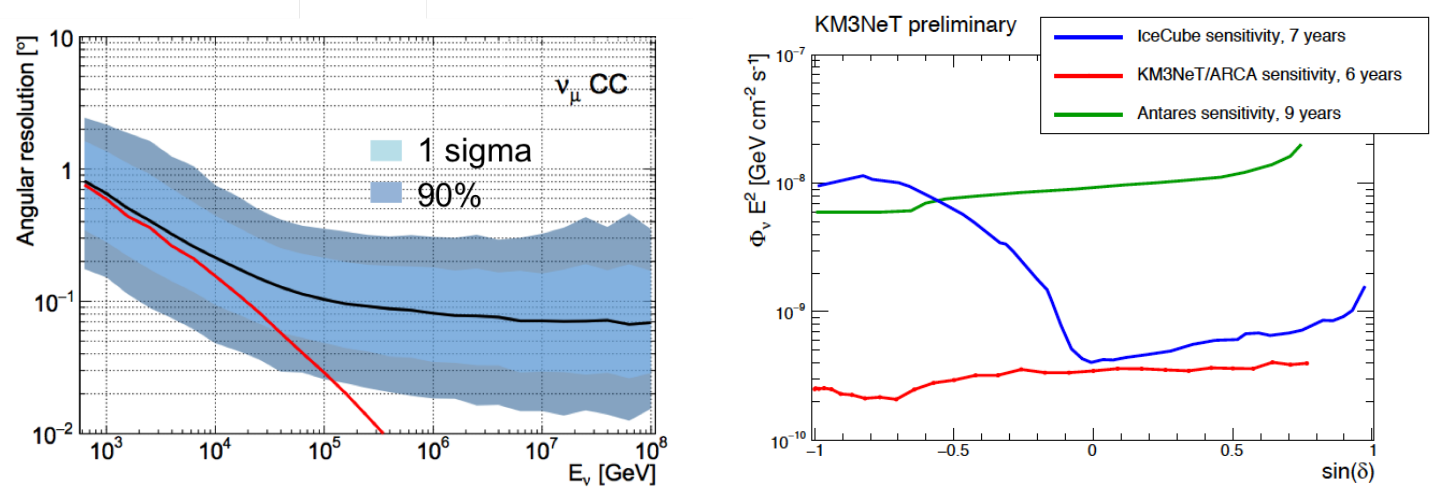

Figure 3: Left panel: median angular error of the reconstructed muon track as function of the neutrino true energy. The coloured bands represent the $68 \%$ and $90 \%$ quantiles. The red line gives the median angle between the true neutrino and muon directions. Right panel: sensitivity for a source with $E^{-2}$ flux as function of the source declination. For comparison the corresponding IceCube [1] and Antares [5] fluxes are shown.

atmospheric muons is on-going. Some preliminary results show a good agreement with simulation and then the functionality of the detector. In its final configuration, KM3NeT/ARCA will consist of 2 building blocks of 115 DUs. With an instrumented volume of about $1 \mathrm{~km}^{3}$, it will offer independent confirmation of the IceCube flux. Furthermore, it will profit of the advantage of this location (better visibility of the Galactic Centre and Galactic Plane compared to the South Pole) and better angular resolution. Optimised to study point-like neutrino fluxes, KM3NeT/ARCA will therefore open the path to identifying their sources.

\section{References}

[1] M. G. Aartsen et al. (IceCube Collaboration), Astrophys. J. 835 (2017) 151.

[2] S. Adrián-Martínez et al. (KM3NeT Collaboration), J. Phys. G: Nucl. Part. Phys. 43 (2016) 084001.

[3] S. Adrián-Martínez et al. (KM3NeT Collaboration), Eur. Phys. J. C 74 (2014) 3056.

[4] S. Adrián-Martínez et al. (KM3NeT Collaboration), Eur. Phys. J. C 76 (2016) 54.

[5] A. Albert et al. (ANTARES Collaboration), arXiv:1706.01857.

[6] K. Melis for the KM3NeT Collaboration, proc. of the 35th International Cosmic Ray Conference 2017, Bexco, Busan, Korea, PoS(ICRC2017)1059.

[7] M. Jongen for the KM3NeT Collaboration, proc. of the 35th International Cosmic Ray Conference 2017, Bexco, Busan, Korea, PoS(ICRC2017)1018.

[8] S. Adrián-Martínez et al. (NEMO Collaboration), Eur. Phys. J. C 76 (2016) 1.

[9] K. Melis, A. Heijboer, M. de Jong for the KM3NeT Collaboration, proc. of the 35th International Cosmic Ray Conference 2017, Bexco, Busan, Korea, PoS(ICRC2017)950.

[10] R. Coniglione et al. for the KM3NeT Collaboration, proc. of the 35th International Cosmic Ray Conference 2017, Bexco, Busan, Korea, PoS(ICRC2017)998.

[11] A. Trovato et al. for the KM3NeT Collaboration, proc. of the 35th International Cosmic Ray Conference 2017, Bexco, Busan, Korea, PoS(ICRC2017)999. 